
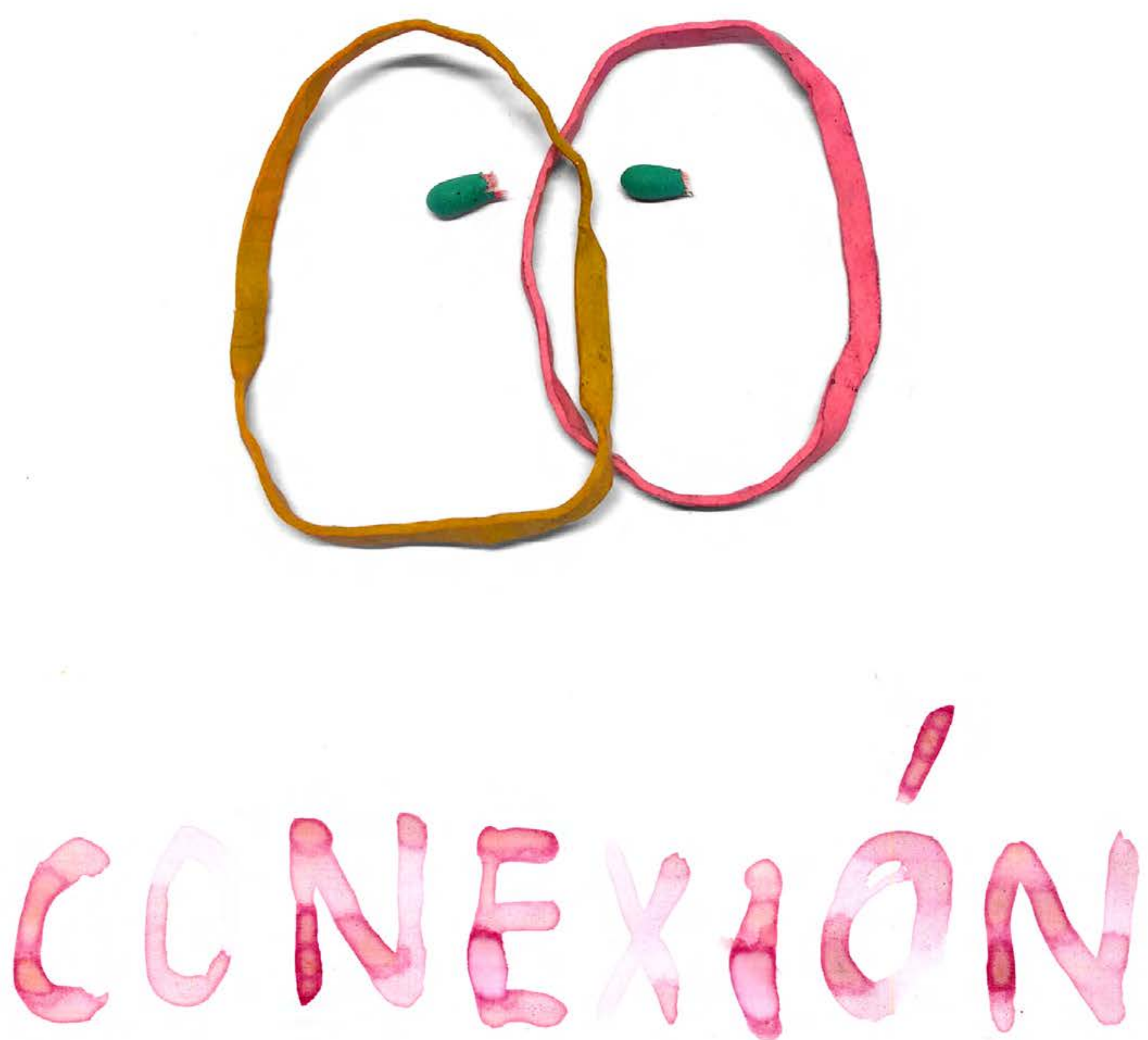


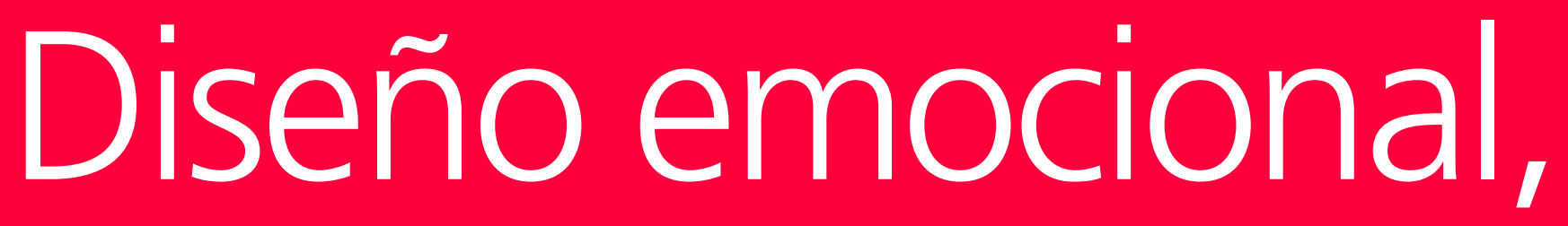

una contribución

a la sostenibilidad

Soler Guitián Andrea 


\section{Diseño emocional, una contribución a la sostenibilidad}

\section{Soler Guitián Andrea}

andreasolerg@gmail.com | Profesora, Universidad Iberoamericana campus Santa Fe y Puebla.

\section{Resumen}

Este artículo aborda el diseño emocional como una contribución a la sostenibilidad, a partir de la generación de relaciones duraderas entre las personas y los productos de diseño. Entendemos al diseño emocional como el enfoque que estudia los estados afectivos que provocan los productos en las personas. Se realiza una revisión del análisis de ciclo de vida del producto, para ejemplificar cómo, al considerar a la afectividad y las emociones, se puede apoyar al diseño sostenible. Además, se presenta el desarrollo de una propuesta de diseño que aplica el conocimiento teórico; la solución tiene el fin de estimular la liga afectiva persona-producto.

Palabras clave | diseño emocional; diseño sostenible; ciclo de vida del producto; afectividad. 
DISEÑO EMOCIONAL, UNA CONTRIBUCIÓN

A LA SOSTENIBILIDAD

\section{Abstract}

This article describes emotional design as a contribution to sustainability through the creation of durable relationships between people and their products. Emotional design is understood as an approach that studies the affective states that products provoke in people. A review of the product life cycle analysis is performed, to show how emotional and affective design can subscribe to sustainable design. In addition, the article shows the development of a design solution that implements theoretical knowledge; the solution aims to stimulate a bond between person-product interactions.

Keywords | emotional design; sustainable design; product life cycle; affect. 


\section{Introducción}

Los objetos forman parte de lo que somos como personas. A través de ellos, reflejamos nuestra identidad (Belk, 1988), describimos quiénes somos y nos ayudan a desarrollar habilidades, por ejemplo, los juguetes estimulan la destreza y aprendizaje de los infantes (Csikszentmihalyi \& Rochberg-Halton, 1981). A través de ellos también se moldean roles sociales, gustos y preferencias. Los productos que utilizamos nos emocionan en diferentes niveles y maneras. Desde el diseño se pueden entender estas emociones y despertarlas, a través de la configuración formal del objeto, su función instrumental, o los significados que asignamos y lo que nos hacen pensar.

A lo largo de la vida de las personas, la relación que tienen con sus objetos cambia y, por tanto, su función y significado también lo hacen. En el libro The meaning of things (1981), Csikszentmihalyi y Rochberg-Halton identifican que los objetos importantes o significativos cumplen diferentes roles para las personas, los cuales están influenciados por la edad y el género. De tal manera, los autores establecen que los niños y jóvenes atribuyen significado a objetos con los que realizan actividades cotidianas, como instrumentos musicales, accesorios deportivos o vehículos; estos son objetos que implican movimiento y al manipularlos adquieren el significado emocional. Por ejemplo, para un adolescente el vínculo con una guitarra sería a partir del uso, las ideas y sueños que este objeto desata; no lo que es, sino lo que puede hacer con él: eso es lo que genera el vínculo afectivo.

Por otra parte, proponen que las personas adultas se identifican con objetos de carácter contemplativo, como libros, objetos decorativos o fotografías, objetos que guardan recuerdos y vínculos con historias de vida. Bajo la misma idea, el adolescente, al crecer, recordará los momentos que pasó con su guitarra y esto será lo que desata las emociones, aunque ya no utilice el objeto.

Para que una persona establezca una relación profunda con un objeto particular debe ser significativo para el ser, si este pierde sentido para el usuario entonces la relación se termina. Es relevante considerar que el sistema de consumo tiende a generar relaciones cortas y sin 
DISEÑO EMOCIONAL, UNA CONTRIBUCIÓN

A LA SOSTENIBILIDAD

sentido con los objetos, son productos desechables. Estimular el consumo permanentemente puede parecer lógico desde una perspectiva económica pero no humana. Mencionamos lo anterior porque de acuerdo con Jonathan Chapman, el consumo de recursos naturales no es proporcional al incremento poblacional; Chapman señala que en los últimos cincuenta años la población mundial se incrementó en un 50\%, pero el uso de recursos naturales aumentó un $1000 \%$. Aunque el incremento poblacional implica un incremento del consumo de recursos, el problema está en la forma poco sostenible, o insostenible en la que se desarrolla el diseño, la manufactura y consumo de objetos en el mundo moderno (Chapman, 2005, 3). Además, de acuerdo a diferentes estimaciones, se ha planteado que el $90 \%$ de los recursos consumidos se transforman en residuos y tan sólo un 10 \% se convierte en bienes provechosos para las personas, que, tras su vida útil y generalmente corta, se convertirán en nuevos residuos (Del Val, 2009). Los datos anteriores muestran por un lado que muchos recursos se desechan casi de forma inmediata, por ejemplo, empaques y por otro que la vida útil de los productos duraderos está disminuyendo. Por ejemplo, en México se desechan 125 mil toneladas de aparatos eléctricos y electrónicos, de los cuales el treinta por ciento son celulares que se utilizan sólo por un par de años (Sánchez-Jimenez, 2017). Estos productos con vidas cortas se crearon a partir de recursos sobre explotados y energía proveniente de la tierra. La idea de crear una infinidad de productos, que estimulen el consumo y a su vez el desecho a partir de recursos que dependen de un planeta finito, es insostenible (Schumacher, 2011).

Este artículo aborda la idea de sostenibilidad a partir de las relaciones emocionales, en particular la durabilidad afectiva. Lo anterior bajo el planteamiento de que para que un diseño tenga dicha cualidad, además de pensar en los procesos de producción y materiales, es también básico pensar en el rol afectivo que las personas desarrollan con los productos, por ejemplo, a través de las emociones y el significado que atribuyen a ellos. Para John Thackara la sostenibilidad significa diseñar para que la gente tenga nuevamente control de las situaciones, plantea un escenario que se basa menos en productos y mercancías y más en las personas $(2013,24)$. Poner atención en esto durante el proceso de diseño se convierte en central para apuntar a que se generen relaciones durables que pospongan el descarte de los objetos. 


\section{La afectividad y el diseño}

Los estados afectivos están presentes en la interacción persona-producto de diferentes formas. Para Pieter Desmet, la respuesta emocional puede ser expresada o provocada por el producto $(2002,19)$. Lo anterior puede lograrse de diferentes maneras, la más sencilla es expresar la emoción a través de la configuración formal; el diseñador utiliza elementos literales asociados con determinado sentimiento. La expresión a la que Desmet hace referencia, puede implementarse a partir de referencias antropomórficas (DiSalvo \& Gemperle, 2003); una estrategia básica y efectiva del diseño para expresar emociones, la podemos encontrar en el uso de emoticones en mensajes de texto, o los diseños de la compañía italiana Alessi, los cuales exploran un elemento de la afectividad a través de metáforas, recurriendo también a colores y texturas. Una cara sonriente expresa alegría y por lo tanto estos objetos pretenden ser alegres y amigables.

Por otra parte, cuando Desmet se refiere a emociones provocadas por los productos la situación es diferente. En este caso no es el objeto el que expresa la emoción, sino que éste incita la actividad o experiencia que provoca la respuesta emocional. Por ello, provocar emociones implica estrategias más complejas de diseño, pues las emociones no se reducen a conductas (Jacob-Dazarola, Ortíz-Nicolás, \& Cárdenas-Bayona, 2016). Tanto cuando hablamos de emociones expresadas, como emociones provocadas necesariamente se establece una relación y hay una experiencia con el producto.

En ambos casos, el de la expresión y la provocación, existe la posibilidad de que la interpretación del usuario sea diferente a la intención del diseñador, o tal vez que los símbolos no signifiquen nada para la persona, o no los puede interpretar; entonces la expresión formal no tendría ningún significado y no habría respuesta emocional. 
DISEÑO EMOCIONAL, UNA CONTRIBUCIÓN

A LA SOSTENIBILIDAD

\section{El factor experiencial y afectivo en el diseño}

Al hablar de emociones provocadas por productos podemos decir que el significado está influenciado por experiencias sensoriales (Gendlin, 1997). En diseño esta experiencia sensorial depende del uso del producto y se refiere a todas las posibles experiencias afectivas involucradas en la interacción entre personas y objetos (Desmet \& Hekkert, 2007). Es importante señalar, que la experiencia abarca otros factores que van más allá de los sentidos; Investigación reciente en experiencia de usuario (Ortíz-Nicolás \& Aurisicchio, 2011) ha identificado cuatro factores que inciden en la experiencia: la persona (biografía, personalidad, habilidades, afectividad, valores o motivaciones), el objeto (forma, textura, uso o marca), la interacción (tarea, actividad) y el contexto (físico, social, sistémico, cultural, situacional y temporal).

La experiencia es dinámica, por ejemplo, al iniciar la primera relación entre la persona y el objeto los sentidos juegan un rol importante, algo que ha sido catalogado como factor visceral (Norman, 2004). La primera impresión se genera a partir de las cualidades sensoriales que las personas perciben de los productos, como el olor de un auto nuevo, la forma de una taza entre las manos o el sonido al abrir una botella de vino. Estas experiencias sensoriales pueden desencadenar una respuesta emocional. También se utiliza el sistema motor para operar los objetos y comunicarnos con ellos (ver Hassenzahl, 2010); durante la interacción los usuarios procesan la información que perciben, interpretan símbolos diseñados estratégicamente (a veces de forma exitosa, otras no) para establecer una relación con el producto. Esta experiencia también está influenciada por el contexto físico como el lugar, ambiente, temperatura, hora del día o la iluminación; y por el contexto temporal que incluye experiencias previas y asociaciones (Desmet \& Hekkert, 2007; Ortíz- Nicolás \& Aurisicchio, 2011). No es lo mismo cuando se utiliza el objeto por primera vez, que cuando se reconocen todas sus fallas o virtudes; tampoco la experiencia es igual cuando el objeto provoca malos recuerdos o, por el contrario, es vinculado con momentos de felicidad.

La persona, la interacción, el artefacto y el contexto determinan la experiencia, la cual es influenciada por las emociones que la persona siente como resultado de la interacción persona-producto. En una línea similar, Hekkert y Shifferstein $(2008,1)$ proponen que la expe- 
DISEÑO EMOCIONAL, UNA CONTRIBUCIÓN

A LA SOSTENIBILIDAD

riencia del producto es resultado de la interacción y depende de experiencias subjetivas. Las personas pueden experimentar una o más emociones, a partir de las cuales se genera una evaluación afectiva del producto. Durante esta valoración, los objetos cobran nuevos significados; las personas establecen relaciones con ellos, algunas insignificantes y otras profundas y duraderas. Cuando las relaciones son duraderas se crean fuertes vínculos afectivos que provocan la creación de significados profundos, a partir de los cuales el objeto cambia, se convierte en el favorito (Mugge, 2007; Chapman, 2005; Ortíz-Nicolás et al., 2013).

\section{Apego con los productos como factor sostenible}

El apego implica una fuerte relación entre un individuo y un objeto en particular, y este vínculo es diferente al que se establece con otros objetos, son posesiones. Es un proceso que se construye a través de tiempo y como resultado de la experiencia. Para que exista apego, es necesario que el objeto provoque algo extraordinario en el individuo y provocan una o varias emociones, las cuales suelen ser positivas (Mugge, 2007, 12; Ortíz-Nicolás et al., 2013). Así, Mugge propone nueve condiciones para estimular el apego entre las personas y los productos, las cuales engloba en cuatro grandes grupos $(2007,28)$ :

1 Expresión personal (el producto me distingue de los demás).

2 Afiliación a un grupo (el producto me conecta con un grupo).

3 Memorias (recuerdos asociados con el producto).

4 Placer (emociones provocadas por el producto).

A partir de este escenario, se puede distinguir entre la experiencia de apropiación hacia un grupo de productos, o hacia un producto en específico. En el primer caso, el sujeto establece una fuerte relación hacia un mismo tipo de objetos o hacia una marca, por ejemplo, una persona puede comunicar rasgos de su identidad a partir de una categoría de producto: las bicicletas en general; esto no implica que la persona prefiera una en particular, sin embargo, en conjunto las bicicletas representan e influyen su personalidad. Por el contrario, cuando el apego es hacia un objeto en específico, no hay otro con el que se pueda 
DISEÑO EMOCIONAL, UNA CONTRIBUCIÓN

A LA SOSTENIBILIDAD

comparar. Por ejemplo, la autora de este artículo, siente un fuerte vínculo con la cafetera de su abuela, no es que todas las cafeteras sean especiales, ésta es única, con sus abolladuras, rasguños y fallas, tiene un significado personal y se siente completamente apegada a ella por lo que representa una relación especial entre abuela y nieta. En ambos casos, la respuesta afectiva refleja las asociaciones personales, por lo que el objeto (o el grupo de objetos) y su significado se vuelven inseparables.

Csikszentmihalyi y Rochberg-Halton (1981) sugieren que el significado de un objeto apropiado o querido varía con el tiempo, partiendo de lo que se hace con él (presente), hacia lo que hizo con él en el pasado. En un momento, el producto ofrece información personal y en otro habla de las relaciones con otras personas. Además, estos autores identificaron tres factores que inciden en el desarrollo de sentimientos de apego hacia los productos que les transmiten un significado especial, uno es la actividad que provocan (acción), otro la reflexión que estimulan (contemplación); y finalmente la relación temporal que se tiene con el objeto.

Se considera que el apego con los productos se enfoca a la relación entre dos partes: una persona y un objeto; desde el diseño podemos enfocarnos a estimular o provocar (Desmet, 2002) dicha relación a través de factores afectivos. La experiencia de apego modifica la forma en la que las personas interactúan con el objeto. Algunos resultados de este fuerte vínculo afectivo son que la persona cuida el objeto, procura repararlo cuando se rompe y pospone su reemplazo el mayor tiempo posible (Mugge, Schifferstein \& Schoormans, 2006). De tal forma y dado el vínculo establecido, el descarte de estos productos sugiere una separación personal, más que un simple desecho (Ekerdt, 2009; Wallendorf \& Arnould, 1988). Al prolongar la vida útil del producto a través del apego lentifica el ciclo de vida, por tanto, reduce la demanda de recursos naturales y disminuye la cantidad de residuos sólidos que son desechados (Mugge, Schifferstein \& Schoormans, 2010).

La afectividad a través del apego y las emociones juegan un rol muy importante en la relación duradera que las personas desarrollan con sus productos y por esta razón se ha 
DISEÑO EMOCIONAL, UNA CONTRIBUCIÓN

A LA SOSTENIBILIDAD

propuesto como estrategia de diseño sostenible (Chapman, 2005; Mugge, 2007). En este artículo se propone el ciclo de vida de los objetos como guía para descubrir diferentes factores de diseño que puedan ser manipulados a partir de las ideas de afectividad y apego.

\section{Ciclos de producto}

Para conservar los recursos naturales es necesaria tanto la reducción de su consumo, como el aumento de la eficiencia en su transformación en bienes utilizables (Del Val, 2009). Para esto, es fundamental el desarrollo de prácticas que permitan su máximo aprovechamiento. Bajo estas ideas, la siguiente sección presenta diferentes estrategias de diseño a partir de los ciclos que se han propuesto a nivel teórico para entender el consumo, la vida y el diseño de productos. El objetivo es presentar un esquema que pueda utilizarse para el diseño emocional sostenible.

\section{Ciclo de consumo del producto}

De acuerdo con el ciclo de consumo (ver Figura 1) la relación entre una persona y un objeto comienza con la adquisición del producto, pasa posteriormente al uso y finalmente termina con el desecho (Mugge, 2008, 325). Este sistema presenta un consumo poco sostenible, porque promueve el desecho y la falta de empatía con los objetos. Se ha sugerido, además que el desperdicio es síntoma de una relación fallida entre el usuario y el objeto (Chapman, 2005,20 ). Esta relación fallida es uno de los factores que propicia el consumismo, mientras menos tiempo un producto esté operando, más pronto se adquiere uno nuevo, se mantiene el consumo y por tanto, el propio sistema de mercado (Joseph, 2011).

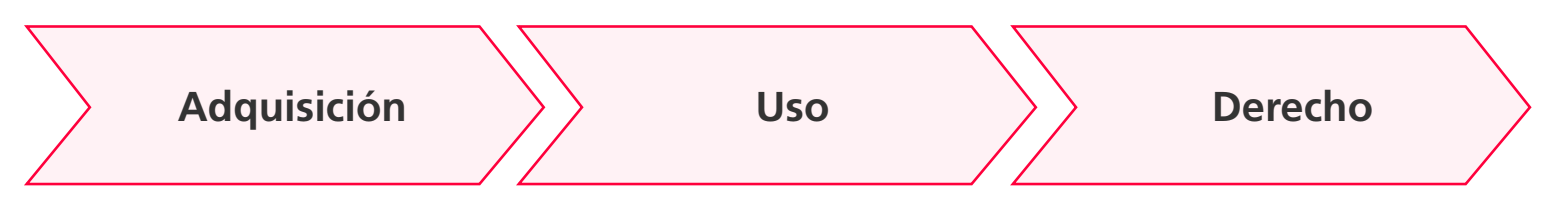

Figura1. Ciclo de consumo. (Elaboración propia). 
DISEÑO EMOCIONAL, UNA CONTRIBUCIÓN

A LA SOSTENIBILIDAD

Si analizamos el ciclo de consumo desde la perspectiva del apego, se identifica que más que un sistema cíclico, funciona de forma lineal y secuencial. Se desechan objetos que todavía sirven y en consecuencia, la relación sujeto-objeto se termina, aun cuando ésta podría ser más duradera.

Con los objetos que estimulan apego existen relaciones estables y duraderas. Por ejemplo, hay productos que son atesorados como piezas de museos, algunos adquieren un valor simbólico que excede su papel funcional, un ejemplo puede ser una vajilla, la almacenamos por años esperando un día especial para ser usada. Además, en las relaciones durables muchas veces el objeto no termina en la basura (ej. juguetes de la infancia) o al menos no de forma casi inmediata. Lo anterior reitera la importancia de considerar el apego en el diseño sostenible.

\section{Ciclo de vida del producto}

Para entender mejor los productos, e ir más allá de su papel como objetos de consumo, se presenta el ciclo de vida, Figura 2. Una vez más Este esquema se utiliza como punto de partida para entender la historia de los productos desde su origen:

$\sum$ Material $\gg$ Producción $\left.\gg \begin{array}{c}\text { Empaque } \\ \text { distribución }\end{array}\right\rangle$ Adquisición $\rangle \geqslant$ Usosecho $\rangle$

Figura 2. Ciclo de vida de los productos. (Elaboración propia).

Tanto el ciclo de consumo como el de vida de los productos (ver Figura 1 y 2) contemplan la adquisición del producto, el uso y el desecho; Una diferencia es que en el segundo se establecen otras etapas, en las que no participa el usuario primario, como son la extracción o transformación del material, la producción, así como el empaque y la distribución. Así, tenemos un ciclo que describe al usuario como consumidor de productos (ciclo de consumo), y otro que se refiere tanto al usuario como al objeto (ciclo de vida). Si al ciclo de vida se agre- 
ga el proceso de diseño, entonces involucra también al diseñador (ver Figura 3). Al agregar el proceso de diseño se hace evidente el papel del diseñador en esta relación y da lugar a una etapa más para el análisis de las relaciones durables, este esquema se plantea como una estrategia para el desarrollo de diseño emocional sustentable. El rol del diseñador es, en gran medida, el centro de varias ideas en torno al diseño sostenible (ver Thackara, 2013) y por lo anterior, su práctica ha sido cuestionada por una falta de autocrítica en relación a la generación de objetos que solo estimulan el consumo (Papanek, 1985; Whiteley, 1993).

$\left.\sum \begin{array}{c}\text { Proceso } \\ \text { de diseño }\end{array}\right\rangle$ Material $\gg$ Producción $\left.\gg \begin{array}{c}\text { Empaque } \\ \text { distribución }\end{array}\right\rangle$ Adquisición $\rangle \geqslant$ Uso $\rangle$

Figura 3. Ciclo de consumo + ciclo de vida +

Durante el proceso de diseño se pueden (o deben) tomar decisiones que impulsen acciones que nos lleven a la sostenibilidad, el diseñador tiene la oportunidad de pensar y definir cómo lograrlo. En este caso, proponemos que puede estimularse el apego como estrategia de sostenibilidad a partir de las diferentes etapas del ciclo completo (ver Figura 3). Lo anterior, con el fin de diseñar productos que tengan una vida útil, larga y duradera.

Investigación previa ha sugerido diferentes soluciones para estimular el apego que los consumidores experimentan con sus productos (Mugge, Schifferstein \& Schoormans, 2010). Por una parte, se propone implementar productos con una utilidad o apariencia superior, de forma tal que el usuario identifique el objeto como diferente o especial. También se puede estimular a partir de la formación de recuerdos o memorias relacionados con el producto, esto a través de la publicidad o marketing; si un producto se asocia con recuerdos, éste gana un significado simbólico especial. Y finalmente proponen estimular el apego mediante el uso de materiales que envejezcan con gracia, las personas asociarán el desgaste del objeto con ciertas memorias (279), como por ejemplo una chamarra de cuero (Chapman, 2005). 


\section{El ciclo completo y el diseño afectivo}

A continuación, vamos a profundizar en cada etapa de la relación con los productos haciendo un análisis de las posibilidades del uso de la afectividad en el diseño de productos. Se presentan ejemplos para cada una de las etapas del ciclo completo (ver Figura 3.), los cuales muestran diferentes ideas y soluciones de diseño orientadas a la sostenibilidad.

\section{Etapa 1: Proceso de diseño}

Proyecto de amor (Love Project), realizado por el diseñador brasileño Guto Requena (2017), plantea un escenario en el que los productos se forman a partir de historias personales. Utilizando tres sensores, uno que lee la actividad cerebral, un sensor de ritmo cardíaco y uno de voz, se recolectan datos de las respuestas físicas y emocionales de un grupo de personas mientras narran una historia de amor. Un software interpreta estos cambios emocionales y los traduce en información para que una impresora 3D genere un objeto.

Cada objeto es único, ya que está determinado por las respuestas emocionales de las personas, los usuarios son parte del proceso de diseño y se involucran con el objeto desde su creación, generando un vínculo afectivo.
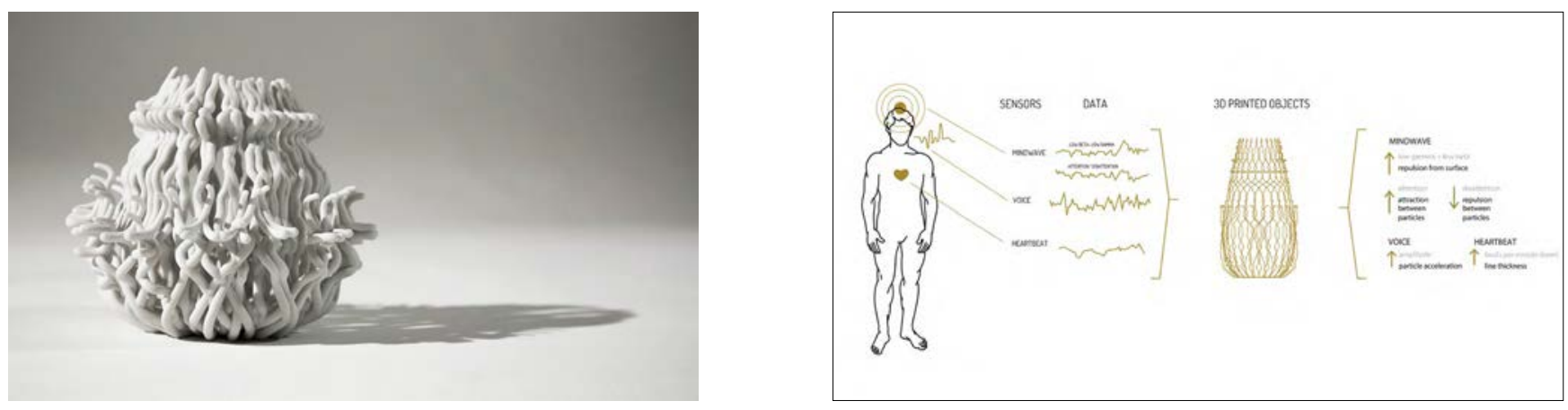

Figura 4. Love Project imágenes de Otavio Whately Pacheco 
Es significativo señalar que en el campo del diseño y las emociones se han generado métodos más estructurados que buscan apoyar al diseñador a incorporar las emociones durante el proceso de diseño (ver Desmet \& Dijkhuis, 2003; Demir et al., 2009; Ortíz-Nicolás et al., 2013) y otros al considerar las diferentes etapas del ciclo de interrelación persona-producto (Jacob-Dazarola \& Torán, 2012).

\section{Etapa 2: Material}

Se ejemplifica con Recuérdame (Rememberme) de Tobias Juretzek (2017). Este proyecto propone una familia de mobiliario: silla y mesa, construidos con ropa vieja como materia prima.

El mobiliario tiene la intención de contener y dar un nuevo significado a objetos olvidados en armarios y cajas. En este caso se utiliza ropa para moldear una silla o una mesa y el resultado es una pieza única, que se logra a través de la materia prima. Los objetos obtienen su propia expresión potenciando el vínculo emocional preexistente con el material.
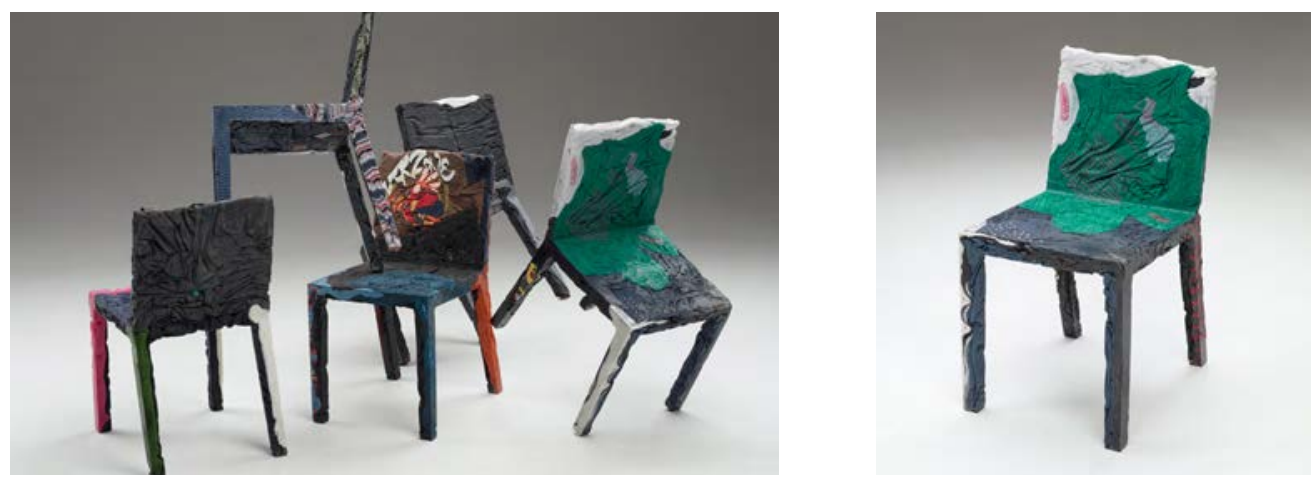

Figura 5. Rememberme. Imágenes de Tobias Juretzek. 
Lo relevante de este proyecto como propuesta de diseño emocional es el uso de la materia prima: la ropa tiene un significado para el usuario, por tanto, tiene una historia que puede ser compartida cada vez que alguien se siente en ella. Para el autor "el diseño se trata de aprecio, de la relación, la separación y la reinvención continua... esto hace que la ropa sea algo valioso" (Juretzek, 2017).

\section{Etapa 3: Producción}

La impresora 3d Prusa 13, diseñada por Josef Průša (2015), basada en los principios del Open Source y RepRap.

RepRap se basa en la fabricación de máquinas auto-replicantes disponibles libremente para el beneficio de las personas que quieran crear sus propios objetos o utilizar los que otros usuarios han diseñado. Al involucrar al usuario en el proceso de producción, tanto en la construcción de maquinarias como en la creación de objetos, la relación entre sujeto y objeto se potencia. Este producto, al igual que otras impresoras 3D, promueve la expresión y satisfacción personal (Mugge, 2007), es una herramienta generada con los principios del código abierto que busca empoderar al usuario, quien ahora tiene el control tanto de lo que crea, como de su propia máquina. De esta forma se propone una forma distinta de producción, estableciendo otros vínculos afectivos, más duraderos.
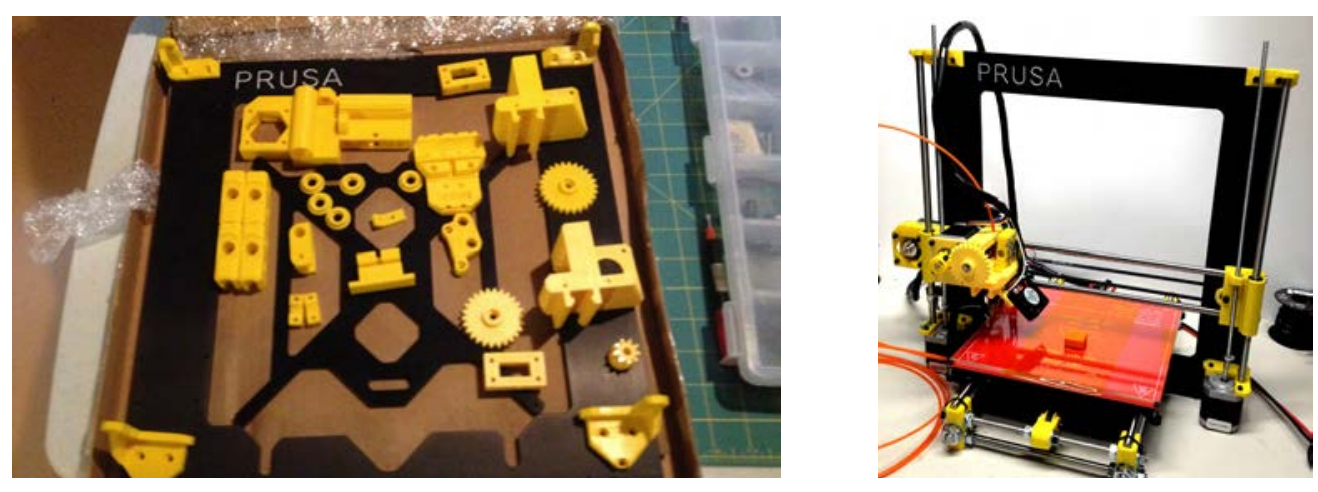

Figura 6. Prusa 13. Imágenes de John Abella. 


\section{Etapa 4: Empaque y distribución}

Se presenta La pequeña bolsa ingeniosa (Clever little bag) diseñada para la marca Puma por Yves Béhar y Fuseproject. El diseño es un empaque sustentable basado en la evolución de la caja tradicional de tenis.

Después de analizar el proceso de fabricación de las cajas tradicionales de zapatos, Yves Béhar crea una solución que responde a la funcionalidad del empaque de manera sostenible, tanto en los procesos de producción, como en la generación de otros usos más allá de su función principal. El 'empaque' de zapatos puede ser utilizado posteriormente como bolsa alargando su vida útil. Esta propuesta evita que el empaque sea desechado inmediatamente al darle un nuevo uso, por tanto, esta solución se alinea con principios de diseño sostenible y emocionalmente durable.

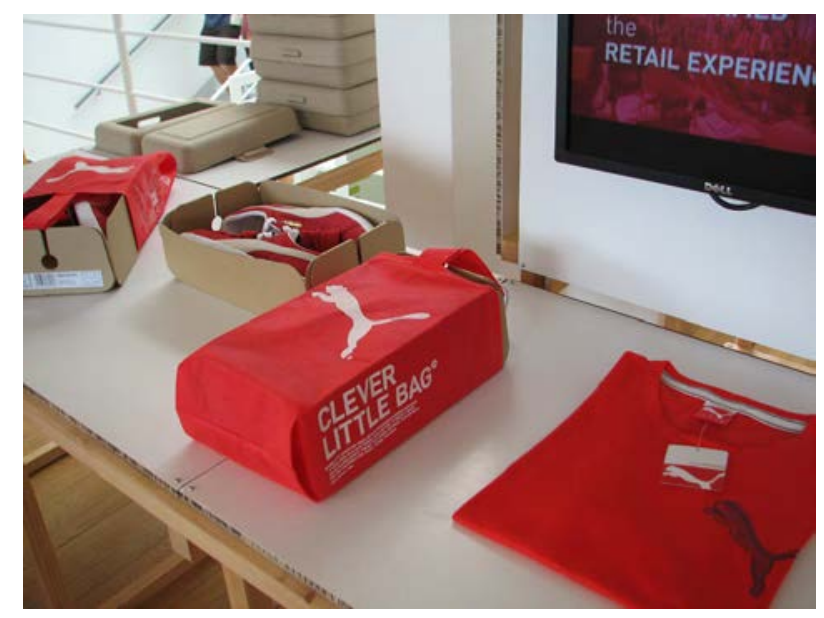

\section{Etapa 5: Adquisición}

Se ejemplifica con Florero rojo y blanco (Red and white base) de Hella Jongerius (1997). Estas vasijas de cerámica son piezas únicas producidas en serie, que cuestionan valores actuales como la perfección. La propuesta formal le da valor a la imperfección, (Ostuzzi, Salvia \& Rognoli, 2011) en un intento de la diseñadora por buscar la belleza en detalles que suelen ser removidos de las piezas industriales, utiliza las costuras del proceso de producción como huellas visibles, dándole un valor estético a las imperfecciones y creando piezas 
únicas a partir de un proceso industrial. Al mismo tiempo evidencia el proceso de producción de cada pieza, proporcionando información al usuario sobre la historia del producto. Al adquirir este florero se obtiene una pieza única, considerando que las imperfecciones varían entre productos, y éste se parece más a una pieza de arte que a uno industrializado. Tanto el significado de tener piezas únicas como la apariencia del objeto son medios que estimulan un vínculo afectivo.

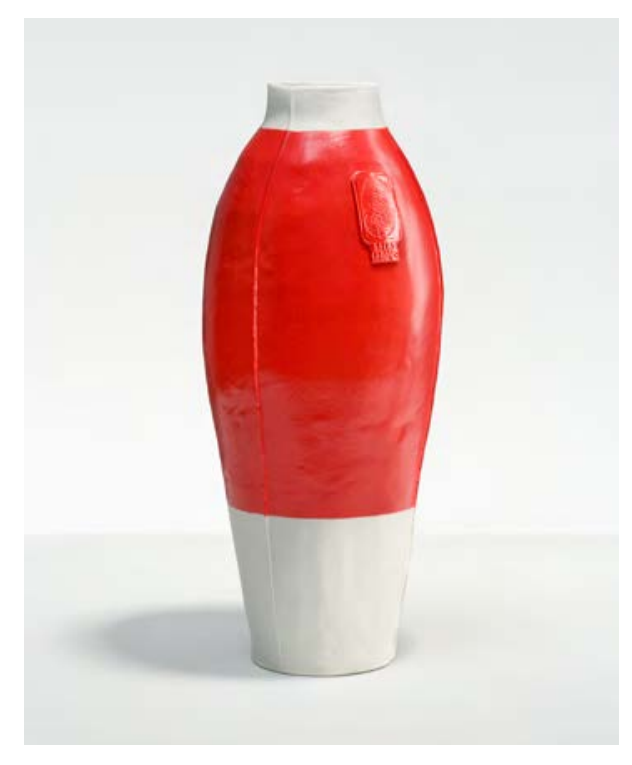

Figura 8. Red and white vase. Imagen de Gerrit Schreurs.

\section{Etapa 6: Uso.}

Se presentan el proyecto Marcas (Stain) de Laura Bethan Wood (2006). Son tazas de cerámica que cambian su apariencia con el uso, se tiñen con el té, revelando poco a poco diferentes patrones que varían según las preferencias personales al tomar la bebida. El objetivo es considerar el paso del tiempo en un producto a través de la apariencia de la taza. 


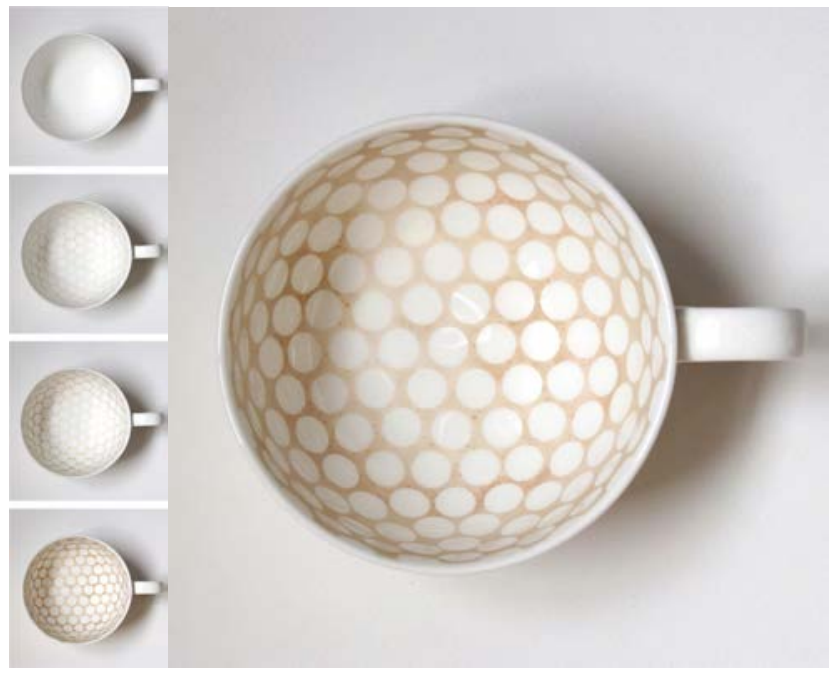

Figura 9. Proyecto stain, el tiempo revela sus marcas. Imágenes de Studio bethanlaurawood.com

En términos generales los productos se hacen menos deseables conforme van envejeciendo (ver Chapman, 2005), si tiene abolladuras, marcas o rayones. En este caso el objeto se transforma con el uso, el paso del tiempo lo hace más bello y personal, como sucede con una cartera de cuero o un escritorio de madera, el material refleja el uso, cuenta historias y esto lo hace más agradable estéticamente. De tal manera, el producto ya no es menos deseable, por el contrario, plantea que los objetos pueden mejorar con el paso del tiempo, generando vínculos y relaciones duraderas con las personas.

\section{Etapa 7: Desecho}

En esta etapa final analizamos el proyecto Buena bolsa (Goedzak) (Waarmakers, 2017). Parte de un producto simple, una bolsa de basura que tiene un objetivo que va más allá de albergar desechos. La idea principal es facilitar el compartir o reutilizar objetos que aún funcionan pero que por alguna razón los dueños planean deshacerse de ellos.

Los participantes reciben las bolsas de plástico y un instructivo. Posteriormente pueden llenar las bolsas y dejarlas en el exterior, como cuando tiran la basura. El color amarillo en las bolsas llama la atención de los transeúntes y la parte transparente revela el contenido, 
los peatones pueden ver los productos y tomar los que deseen. De esta forma se estimula el uso de productos en buen estado que no eran utilizados por sus dueños, evitando el desecho y promoviendo relaciones sostenibles.

El proyecto se ha desarrollado con el apoyo del gobierno en las ciudades de Ámsterdam y Eindhoven. Waarmakers, los creadores de Goedzak, plantean que "un producto es más que material, forma y función; Los productos median nuestra interacción con el mundo. Los productos influyen en nuestro comportamiento, y con eso nuestro medio ambiente"(2017).
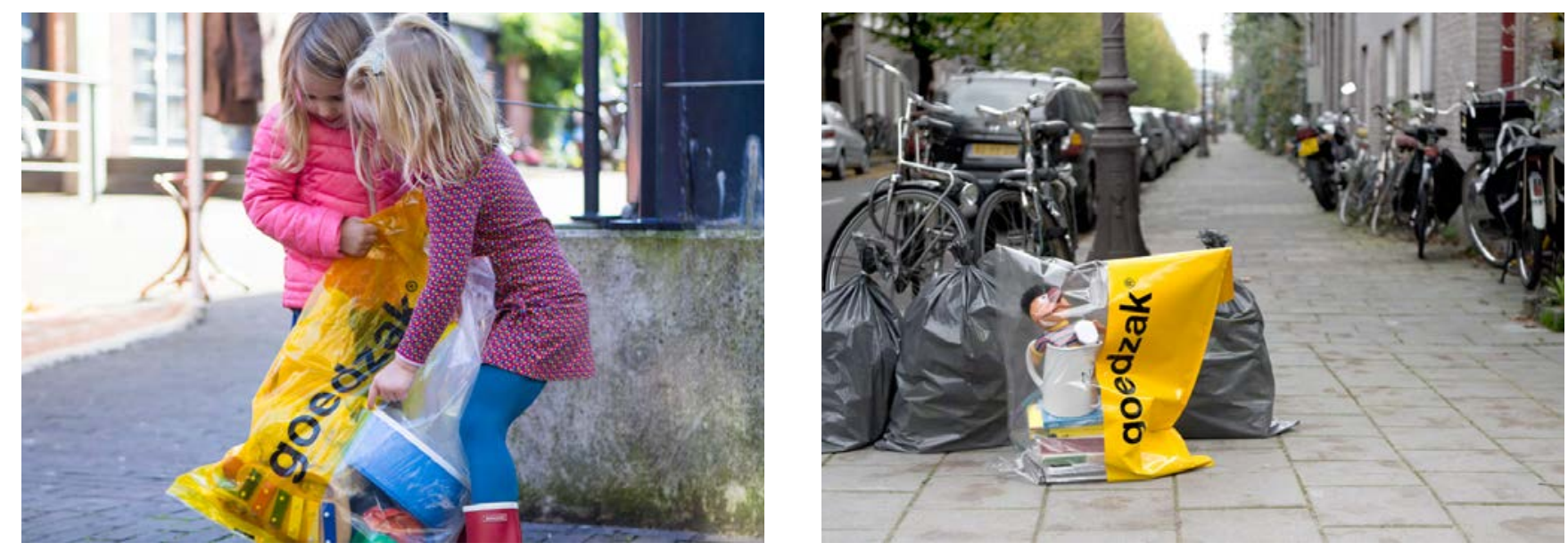

Figura 10. Goedzak, más que una bolsa. Imágenes

recuperadas de http://www.waarmakers.nl/projects/goedzak

\section{Discusión}

Los siete ejemplos que hemos presentado proponen diferentes relaciones entre las personas y los objetos, mediadas por la afectividad y sostenibilidad. El análisis de cada producto está contextualizado en al menos una de las etapas planteadas en el ciclo completo (ver Figura 3.), tratando de resaltar características funcionales, estéticas, productivas o metodológicas, que indican el potencial de los productos en la construcción de relaciones duraderas entre las personas y los productos. Esto evidencia la variedad de posibilidades de diseño enfocado a una perspectiva durable y sostenible. 
Para complementar este trabajo, la Tabla 1 presenta diferentes factores que especialistas en diseño pueden considerar como punto de partida al buscar intencionalmente la generación de soluciones emocionalmente durables, por etapa o considerando el ciclo completo. La tabla es el resultado del análisis realizado para cada etapa y los productos seleccionados, combinado con las ideas de otros autores que se utilizaron como referencia para este artículo (Chapman, 2005; Mugge, 2007).

\begin{tabular}{|c|c|}
\hline Etapa & Factores a considerar \\
\hline $\begin{array}{l}\text { ETAPA } 1 \\
\text { Proceso de diseño }\end{array}$ & $\begin{array}{l}\text { - Creación y uso de métodos de diseño enfocados a las emociones y a la sostenibilidad. } \\
\text { - Análisis emocional de usuarios. (Previo al uso y posteriormente, con el fin de realizar futuras modificaciones } \\
\text { en el diseño) } \\
\text { - Tener como objetivo estimular emociones relevantes en relación al objeto de diseño. }\end{array}$ \\
\hline $\begin{array}{l}\text { ETAPA } 2 \\
\text { Material }\end{array}$ & $\begin{array}{l}\text { - Uso de materiales reusables o reciclables. } \\
\text { - Creación de materiales sostenibles a partir del reusó. } \\
\text { - Materiales duraderos. } \\
\text { - Con significado o carga emocional. } \\
\text { - Materiales que "envejecen bien" (Chapman, 2005) }\end{array}$ \\
\hline $\begin{array}{l}\text { ETAPA } 3 \\
\text { Producción }\end{array}$ & $\begin{array}{l}\text { - Procesos accesibles o abiertos para los usuarios que fomenten el vínculo entre personas. } \\
\text { - Creación de producciones personales que faciliten la carga emocional y significados personales } \\
\text { en los productos. } \\
\text { - Procesos de producción responsables con el medio ambiente y con los productores. } \\
\text { - Soluciones de diseño que hagan eficiente la producción y propicien la reparación del producto. }\end{array}$ \\
\hline $\begin{array}{l}\text { ETAPA } 4 \\
\text { Distribución / Empaque }\end{array}$ & $\begin{array}{l}\text { - Distribución local. } \\
\text { - Empaques sostenibles que promuevan el reúso. } \\
\text { - Evitar lo desechable. }\end{array}$ \\
\hline $\begin{array}{l}\text { ETAPA } 5 \\
\text { Adquisición }\end{array}$ & $\begin{array}{l}\text { - Inversión. } \\
\text { - Promover desde el diseño lo auténtico, único o personal para la creación de significados. } \\
\text { - Objetos que puedan ser heredados, o diseñar a partir de la estimulación de recuerdos como estrategia } \\
\text { para la compra de un producto. }\end{array}$ \\
\hline $\begin{array}{l}\text { ETAPA } 6 \\
\text { Uso }\end{array}$ & $\begin{array}{l}\text { - Diferentes posibilidades de uso. } \\
\text { - Objetos que puedan transformarse según necesidades. } \\
\text { - Creación de conceptos de diseño que provoquen nuevas relaciones, por ejemplo, las impresoras 3D. } \\
\text { - Considerar la personalidad del producto. } \\
\text { - Objetos durables que guarden memorias y recuerdos adheridos con el tiempo. } \\
\text { - Refacciones que faciliten la longevidad del producto. }\end{array}$ \\
\hline $\begin{array}{l}\text { ETAPA } 7 \\
\text { Desecho }\end{array}$ & $\begin{array}{l}\text { - Garantías que promuevan la longevidad del producto. } \\
\text { - Empresas responsables por el desecho de sus productos incluyendo el empaque. } \\
\text { - Diseños creados para la reparación y reciclaje de sus componentes. } \\
\text { - Hábitos y costumbres para el desecho de productos. }\end{array}$ \\
\hline
\end{tabular}

Tabla 1. Ciclo completo y los factores de diseño emocional que pueden considerarse para un diseño sustentable. [Elaboración propia]. 
Los conceptos presentados pueden funcionar como estrategia durante el proceso creativo para generar proyectos enfocados en la durabilidad afectiva. De esta forma el diseño emocional puede considerarse como una herramienta para producir objetos sostenibles, al explorar y promover otras formas de relacionarnos, tanto con los productos que consumimos, como con el entorno.

\section{Ejercicios de diseño basado en las relaciones durables}

Esta sección presenta un proyecto que la autora desarrolló en la Universidad de Trent en Nottingham, Inglaterra. Es una propuesta que busca estimular relaciones durables a través de la afectividad. Considerando el ciclo completo presentado en la Figura 3, el proyecto se inserta en la etapa 3: producción. Para su desarrollo se recurrió a la información presentada en la Tabla 1. La solución es una máquina que lleva por nombre Créame, máquina de emociones (MadeMe, machine of emotions) y tiene como objetivo principal, ilustrar como una solución de diseño orientada al proceso de producción, puede explorar los vínculos afectivos entre las personas y los objetos. Si bien, para esto se diseñó una máquina que utiliza papel de desecho como materia prima, la cual se transforma y moldea en un portavaso, el punto central, es que las personas se involucran directamente en la creación de sus objetos. El concepto de diseño emocionalmente durable (Chapman, 2005), es la base con la que se genera MadeMe al explorar la posibilidad de romper los patrones de consumo hacia unos más sostenibles. A partir de ello, se propone que el usuario genere su propio material, de forma tal que la relación con el objeto sea mucho más larga y empiece antes que en la establecida en el ciclo de consumo (ver Figura 3), en el que no es claro, para los usuarios, de dónde vienen los objetos o cómo fueron producidos. 


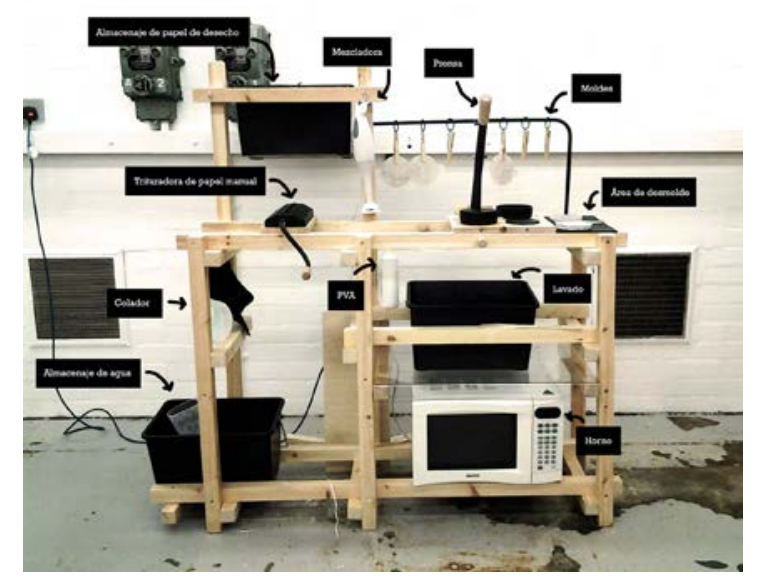

Figuro 11. MadeMe y sus componentes. [Elaboración propia].

La máquina está compuesta por diferentes elementos que guían al usuario durante el proceso de producción. En MadeMe, todo está ordenado para simular un proceso de producción en serie, que avanza de derecha a izquierda y de arriba a abajo. Los electrodomésticos se consiguieron de segunda mano y se utilizaron materiales de reúso para construir la estructura y fabricar las herramientas (ver Soler, 2016).

El proceso para transformar el papel en objetos, incluye seis etapas, cada una determinante para convertir el material de desecho en un objeto de diseño emocionalmente durable. La siguiente figura describe este ciclo:

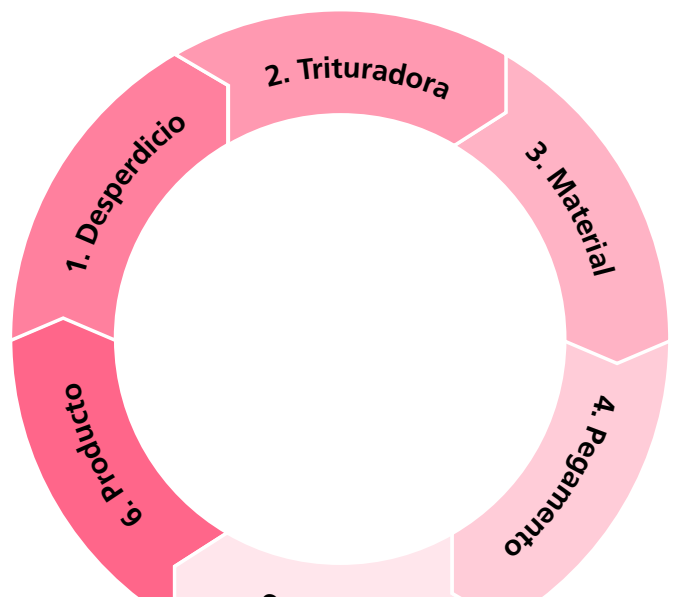

оәріоw ·

Figuro 12. Ciclo de seis etapas para proceso de MadeMe. [Elaboración propia]. 
A partir de estas etapas se genera el producto final, un portavasos con seis opciones, cada una representa una de las emociones básicas planteadas por Paul Ekman (en Benson, Sabbagh, Carlson \& Zelazo, 2012, 197). El uso de las expresiones faciales en el producto tiene la finalidad de que los usuarios se identifiquen con el objeto, más no determinar la emoción, es decir, se utilizan como expresión en vez de provocación.

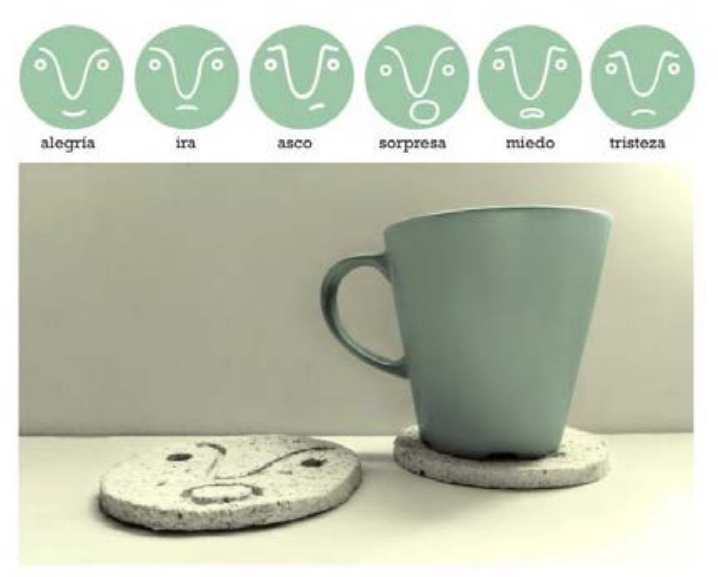

Figuro 13. Expresiones faciales y producto final de MadeMe.

Por otra parte, el uso de desecho como materia prima apela a la idea de que el material está cargado de historias, es decir, ya tuvo una vida, ya se procesó en algún lugar del mundo y se utilizó por alguien más, por lo que tiene una carga emocional desde el inicio; esta idea se alinea con lo que propone Tobias Juretzek en Rememberme. Al reutilizarlo se estaría alargando la vida útil, deja de ser un desecho y se le da un nuevo objetivo.

MadeMe se creó como una máquina de significados, al realizar las seis etapas el usuario vive la experiencia de crear sus propios objetos y se relaciona con ellos, por tanto, se asume que se experimentaran varias emociones durante el proceso; las cuales pueden provocar el vínculo afectivo con el producto.

1 Paul Ekman (1934- ) Psicólogo por la Universidad de Chicago. Realizó su doctorado en sicología clínica en la Universidad Adelphi en Nueva York y posteriormente continuó su investigación en comportamiento no verbal y expresiones faciales en la Universidad de California, San Francisco. Este trabajo introdujo a Ekman en el hasta entonces inexplorado campo de la sicología de las emociones. (Benson, et al., 2012, 197) 
Al involucrar a las personas con los productos desde su creación, se establece una nueva relación, ahora los usuarios conocen su origen, forma de producción, tiempo que lleva producirlo y todo el trabajo que implica. Siguiendo los presupuestos teóricos planteados por Chapman y Mugge, esta información será la base para generar un vínculo emocional duradero con el producto (Chapman, 2005; Mugge, 2007). Haciendo una analogía con las relaciones personales, aquellas personas con las que compartimos lo que somos, son las que permanecen más tiempo a nuestro lado, o con las que establecemos vínculos más duraderos, por tanto, diríamos que MadeMe es una máquina de narrativas que vincula a las personas con los objetos, revelando su historia para provocar relaciones emocionalmente durables.

Las posibilidades de MadeMe como máquina para producir objetos de papel de desecho son varias; se pueden crear diferentes objetos al cambiar el molde, así con una máquina podrían producirse objetos en escuelas, hogares u oficinas. Es una pequeña fábrica de productos, las posibilidades están abiertas y una vez que los usuarios comprendan su funcionamiento, pueden modificar y perfeccionar el proceso de producción. En cierta forma es un resultado similar al de las impresoras 3D, con las que las personas pueden producir sus propios objetos en sus hogares, pero en este caso lo hacen con tecnología simple y con materia prima que proviene de desecho.

\section{Discusión general}

En las últimas décadas se han desarrollado diferentes estrategias y soluciones de diseño sostenible, algunas acertadas, otras no tanto, llegando al punto de utilizarlas como otra estrategia de mercado. A pesar de lo anterior, pensar y diseñar de manera sostenible es una necesidad en la sociedad actual. Este artículo presenta un análisis de la relación con los objetos, desde la perspectiva de la afectividad, basada en el ciclo de vida del producto para generar una estrategia de diseño sostenible. Utiliza ejemplos que ilustran cómo la afectividad puede considerarse en cada etapa y expone una propuesta de diseño, sustentada en teorías de diversos autores, que utiliza el diseño afectivo y emocional. Lo anterior se alinea respecto al rol significativo que tiene la apropiación y las relaciones afectivas durables en relación a la sostenibilidad. 
Utilizar las emociones o los vínculos afectivos como estrategia sostenible no es algo nuevo, todos conservamos objetos heredados o encontramos un nuevo uso a productos desechables. Aquí hemos planteado la posibilidad de que diseñadores, adapten estas soluciones (usualmente desarrolladas por los usuarios) a sus procesos de diseño. Es decir, diseñar con la intención de generar vínculos afectivos que promuevan relaciones durables entre las personas y los objetos, en consecuencia, diseños sostenibles. Con base en el análisis presentado en el ciclo completo del producto delineamos estrategias particulares para lograrlo; además nuestro enfoque trata de insertarse en relación a ideas preexistentes en el diseño sustentable, como la del ciclo de vida del producto, con lo que buscamos conectar de manera más directa las ideas de sostenibilidad y afectividad. Esta mirada y estrategia pueden complementarse con otras ideas similares, como la historia de vida del objeto, propuesta por Martín-Juez (2013) y que describe cinco etapas y acontecimientos comunes en los productos: como producto virtual, en la producción, en los procesos de circulación de las materias primas y del producto fabricado, en el consumo, y finalmente, en el uso y el desecho. Existen también propuestas que consideran las experiencias placenteras y su rol en la sostenibilidad (Ortíz-Nicolás, 2017). Como mencionamos anteriormente, las experiencias placenteras se caracterizan por evocar emociones positivas.

Este artículo argumenta la importancia de considerar la afectividad en el diseño sostenible. Dicho argumento se estructura en dos perspectivas. Por una parte, se plantea una disminución de desperdicio; la mayoría de los objetos son desechados antes de que dejen de funcionar. De acuerdo con Jonathan Chapman, más del noventa por ciento de los recursos naturales se convierten en basura en tan solo tres meses $(2008,8)$, al provocar relaciones duraderas, la vida útil del objeto será más larga y por lo tanto su desecho también se posterga. La segunda perspectiva se basa en las teorías de apropiación identificadas por Ruth Mugge, las cuales pueden ser objetivos del diseñador, con el fin de crear objetos que promuevan las expresiones personales de los usuarios, de la misma forma que nos vinculen con los demás; objetos que sean placenteros para el usuario y guarden recuerdos de lo que somos. Estos productos tendrán mayor significado para quien los use, pero también pueden ser herramientas para promover cambios sociales que beneficien el futuro, desde 
el uso de materiales sostenibles, hasta propuestas de producción independientes que desafíen el sistema de mercado actual (Ver Ortíz-Nicolás, 2016).

Reconociendo que son los usuarios quienes le dan significado a los objetos, consideramos que utilizar estrategias afectivas como herramienta para la sostenibilidad, es una oportunidad para que diseñadores generen objetos de los cuales las personas se sientan orgullosos, productos que quieran conservar y heredar; objetos que evolucionen en el tiempo junto con nosotros. Estos diseños proponen nuevas relaciones, no solo entre sujetos y objetos, también formas sostenibles de relacionarnos entre personas y el planeta. "Los problemas persistirán si no detenemos el ritmo y las modalidades de nuestros modos actuales de producción y de consumo; si no modificamos nuestras maneras de entender el problema" (Martín-Juez, 2013, 8). Este artículo busca contribuir al diseño sostenible al considerar teorías que surgen a partir de la afectividad las cuales ayudan a estructurar ideas potenciales en relación al ciclo de vida del producto. Se hace un esfuerzo por presentar diferentes soluciones para cada una de las etapas, lo que posibilita el uso de diferentes estrategias de acuerdo a las necesidades de los usuarios y características del proyecto de diseño.

Un reto que involucra a ambos enfoques (afectivo y sostenible) es que las sociedades son muy complejas y están en constante cambio, además cada individuo es diferente y los vínculos afectivos son subjetivos; por lo que las propuestas o estrategias de diseño aquí planteadas deben analizarse de acuerdo a los contextos y escenarios, es decir los diseñadores deben darles significado. Este es un gran reto, y puede decirse que el análisis de ciclo de vida del producto es un primer paso, para continuarlo se debe confirmar que las estrategias son efectivas, si bien existe cierta evidencia (Chapman, 2005; Mugge, Schifferstein \& Schoormans, 2010) se deben corroborar en otras latitudes, considerando que existen muchos factores involucrados. Por otra parte, consideramos que esta investigación puede complementarse con otros proyectos y temas relevantes; existen varios factores que hace falta explorar y que los diseñadores no las tomamos mucho en cuenta; por ejemplo, la temporalidad (ver Mugge, 2007; Ortíz-Nicolás \& Aurisicchio, 2011), que apenas aquí hemos indicado. 


\section{Conclusiones}

Es necesario considerar que los productos representan valores y estilos de vida que muchas veces están determinados por el mercado, y que esta situación no es cuestionada por diseñadores o usuarios (ver Whiteley, 1993), consumimos objetos que representan ideas efímeras (pasan de moda) o nos damos cuenta de que en realidad no los necesitábamos, entonces los desechamos, a pesar de que aún sean útiles. Al mismo tiempo, los avances tecnológicos y la forma de vida moderna se mueven cada vez más rápido. Los objetos se vuelven obsoletos casi al momento de adquirirlos, pues siempre se está planeando la versión más reciente todo con el fin de estimular el consumo.

Jonathan Chapman menciona que los productos manufacturados sirven para ilustrar nuestras aspiraciones, al mismo tiempo son uno de los factores que nos definen $(2008,61)$; desde esta idea, consideramos que el diseño emocionalmente durable y la apropiación son una declaración hacia las relaciones modernas. Desde el diseño se puede romper con el ciclo de consumo, al considerar otras formas de relacionarnos con los productos, estas relaciones pueden ser provocadas a través de la interacción con los objetos, siempre y cuando se tengan en cuenta durante el proceso creativo, es decir debe existir una intencionalidad por parte de los especialistas en diseño. 


\section{Referencias}

Belk R.W. (1988). Possessions and the Extended Self, Journal of Consumer Research, 15 (2), 139-168.

Benson J. E., Sabbagh M. A., Carlson S. M., Zelazo P. D. (2012). Individual differences in executive functioning predict preschoolers' improvement from theory-ofmind training. Dev. Psychol. 49 1615-1627 10.1037/ a0031056

Chapman, J. (2005). Emotionally Durable Design. Objects, experiences \& empathy. Abingdon: Earthscan.

Chapman, J. (2008). Sustaining relationships between people and things. En Design and Emotion Moves (59-77). Newcastle: Cambridge Scholars Publishing.

Csikszentmihalyi, M., \& Rochberg- Halton, E. (1981). The meaning of things. Domestic symbols and the self. Cambridge: Cambridge University Press.

Del Val, Alfonso. (2011). El problema de los residuos en la sociedad del bienestar, Boletín $C F+S$, núm. 50, Recuperado de: http://habitat.aq.upm.es/boletin/n50/ aaval.html, 29-38.

\section{Demir, E., Desmet, P. M. A., \& Hekkert, P. (2009).}

Appraisal patterns of emotions in human-product interaction. International Journal of Design, 3 (2), 41-51.

Desmet, P. (2002). Designing Emotions. Delft: TU Delft.

Desmet, P., \& Hekkert, P. (2007). Framework of Product Experience. International Journal of Design 1 (1), 57-66.

Desmet, P., \& Dijkhuis, E. (2003). A wheelchair can be fun: a case of emotion-driven design. Proceedings of the 2003 international conference on Designing pleasurable products and interfaces, 22-27. ACM.

DiSalvo, C., \& Gemperle, F. (2003). From seduction to fulfillment: the use of anthropomorphic form in design. Proceedings of the 2003 International Conference on Designing Pleasurable Products and Interfaces, 67-72. Recuperado de: https://doi. org/10.1145/782896.782913
Ekerdt, D. J. (2009). Dispossession: The Tenacity of Things. En Consumption and generational change. The rise of consumer lifestyles (63-78). Londres, UK: Transaction Publishers.

Fuseproject. (2010). Fuseproject. Recuperado de: https://fuseproject.com/work/puma/clever-little-bag/?focus=overview

Gendlin, E. (1997). Experiencing and the creation of meaning. Illinois: Northwestern University Press.

Hassenzahl, M. (2010). Experience design: Technology for all the right reasons. En Synthesis Lectures on Human-Centered Informatics, 3 (1), 1-95.

Hekkert, P. \& Schifferstein, H. N. (2008). Product Experience. New York: Elsevier.

International, N. (2016). Numatic International Ltd. Recuperado de: https://www.numatic.co.uk

Jack, I. (2010). Can manufacturing fill Britain's economic vacuum? Recuperado de: http://www.theguardian. com/commentisfree/2010/jul/24/can-manufacturing-fill-british-economic-vacuum.

Jacob-Dazarola, R. \& Torán, M. M. (2012). Yeah, I talk to my car...so what?: Roles and levels of closeness in person-product relationship. International Journal of Designed Objects, 6 (3), 49-60.

Jacob-Dazarola, R., Ortíz-Nicolás, J. C. \& Cárdenas-Bayona, L. (2016). Behavioral Measures of Emotion. En Emotion Measurement (101-124). Amsterdam: Elsevier. Recuperado de https://doi.org/10.1016/ B978-0-08-100508-8.00005-

Sanchéz-Jimenez, S. (2017, 06, 25) Aumentan hasta $78 \%$ los residuos electrónicos en el valle de México [versión electrónica]. La Jornada. Recuperado de: http://www.jornada.unam.mx/2017/01/15/sociedad/026n1soc

Jongerius, H. (1997). Big White Pot and Red White Vase. Recuperado de: http://www.jongeriuslab.com/work/ big-white-pot-and-red-white-vase 
Joseph, P. (2011). Zeitgeist: Moving foward [Película].

Juretzec, T. (2017). Rememberme: Tobias Juretzec. Recuperado de: http://www.tobiasjuretzek.com

Martín-Juez, F. (2013). Homoindicadores. Ciudad de México: CIDI-UNAM.

Mugge, R. (2007). Product attachment. Delft: Delft University of Technology.

Mugge, R. (2008). Product Attachment and Satisfaction: The Effects of Pleasure and Memories. European Advances in Consumer Research vol. 8, 325-331.

Mugge, R., Schifferstein, H. N. \& Schoormans, J. P. (2006). A Longitudinal Study of Product Attachment and its Determinants. European Advances in Consumer Research vol.7, 641-647.

Mugge, R., Schifferstein, H. N., \& Schoormans, J. P. (2010). Product attachment and satisfaction: understanding consumers' post-purchase behavior. Journal of Consumer Marketing, vol. 27, 271-282. Recuperado de https://doi.org/10.1108/07363761011038347

Ortíz-Nicolás, J. C. (2017). Pleasant experiences and sustainable design. Routledge Handbook of Sustainable Product Design. Recuperado de https://doi. org/10.4324/9781315693309

Ortíz-Nicolás, J.C. (2016). Diseñando el cambio. La innovación social y sus retos. Economía Creativa, otoño-invierno, vol. 6, 9-35.

Ortíz Nicolás, J. C., Aurisicchio, M., \& Desmet, P. (2013). Designing for anticipation, confidence, and inspiration. Proceedings of the 6th International Conference on Designing Pleasurable Products and Interfaces (31-40). ACM.

Ortíz-Nicolás, J.C. \& Aurisicchio, M (2011). A Scenario of User Experience. En Proceedings of the 18th International Conference on Engineering Design (ICED 11), vol. 7: Human Behaviour in Design, (182-193).
Ostuzzi, F., Salvia, G., \& Rognoli, V. (2011). The value of imperfection in industrial product. Proceedings of the Designing Pleasurable Products and Interfaces (DPPI 2011).

Papanek, V. (1985). Design for the real world. London: Thames and Hudson.

Prusa, J. (2015). Prusa i3 3D Research. Recuperado de: http://shop.prusa3d.com/en/

Requena, G. (2017). Love project. Recuperado de: https://gutorequena.com/love-project/

Schifferstein, H. N. J., \& Zwartkruis-Pelgrim, E. P. H. (2008). Consumer-Product Attachment: Measurement and Design Implications. International Journal, 2(3), 1-13.

Schumacher, E. F. (2011). Lo pequeño es hermoso. Madrid: Ediciones AKAL.

Sirgy, M. J., Grewal, D. and Mangleburg, T. (2000) Retail Environment, Self-Congruity, and Retail Patronage: An Integrative Model and a Research Agenda, Journal of Business Research vol. 49 127-138.

Soler, A. (2016). Emociones y diseño. Tesis de maestría. Universidad Nacional Autónoma de México.

Thackara, J. (2013). Diseñando para un mundo complejo. México: Designio.

Wallendorf, M., \& Arnould, E. J. (1988). My Favorite Things: A cross-culftural inquiry into object attachment, possessiveness, and social linkage. Journal of Consumer Research, vol. 14, 531-547. Recuperado de https://doi.org/10.1300/J136v02n01_11

Waarmakers. (2017). GOEDZAK IS NOT (JUST) A BAG. Recuperado de: waarmakers.nl/projects/goedzak/

Wood, L. B. (2006). Stain 2016. Recuperado de: http://www.bethanlaurawood.com/work/stain/

Whiteley, N. (1993). Design for society. London: Reaktion books. 